\title{
Twelve-Year Follow-up After Peptide Receptor Radionuclide Therapy
}

\author{
Michael Gabriel ${ }^{1,2}$, Bernhard Nilica ${ }^{1}$, Bernhard Kaiser $^{2}$, and Irene J. Virgolini ${ }^{1}$ \\ ${ }^{1}$ Department of Nuclear Medicine, Medical University of Innsbruck, Innsbruck, Austria; and ${ }^{2}$ Institute of Nuclear Medicine and \\ Endocrinology, Kepler University Hospital, Linz, Austria
}

Peptide receptor radionuclide therapy (PRRT) has been used for more than 20 y as a systemic treatment approach in inoperable or metastatic somatostatin receptor-positive tumors. The purpose of this study was to analyze the long-term outcome of PRRT with regard to the most commonly used radiopharmaceuticals, ${ }^{90} \mathrm{Y}$ DOTATOC and ${ }^{177}$ Lu-DOTATATE. Methods: This retrospective clinical study included a total of 44 consecutive patients $(27$ men) with advanced tumors and enhanced somatostatin receptor expression. Mean age at initial diagnosis was 60 y (SD, 11.3 y; range, 40-84 y). Median follow-up was 80 mo. For ${ }^{177}$ Lu-PRRT, the mean number of cycles administered was $5.3 \pm 2.5$ and the mean activity was $27.2 \pm$ $14.9 \mathrm{GBq}$ per patient. For ${ }^{90} \mathrm{Y}-\mathrm{PRRT}$, the mean number of cycles administered was $5.5 \pm 2.6$ and the mean activity was $14.7 \pm 7.3$ GBq per patient. Overall, 378 cycles were administered (mean, $8.6 \pm$ 3.4 cycles per patient), with an overall cumulative activity of $1,514.1$ GBq. Results: Median overall survival was 79 mo. Twenty-one $(77.8 \%)$ of the 27 men and $9(52.9 \%)$ of the 17 women had died $12 \mathrm{y}$ after commencement of PRRT. The shortest duration of illness was $8 \mathrm{mo}$ and the longest $155 \mathrm{mo}$. Severe side effects (World Health Organization grades III and IV) were seen in 9 of the 14 patients still alive. Chronic kidney disease in combination with anemia was the most common finding in the 9 patients with severe side effects. A poor prognosis was found for those patients who showed progressive disease, in comparison with patients with cumulative disease control after initial PRRT (log rank, $P<0.001$ ), whereas women and patients with no more than 2 tumor sites seemed to especially benefit from PRRT (not reaching significance levels). Conclusion: PRRT is encouraging in terms of long-term outcome. Thirty-two percent (14/44 patients) of the patients with metastatic or inoperable disease were still alive more than $12 \mathrm{y}$ after the beginning of radionuclide therapy. Possible predictors for favorable outcome are having an initial response to PRRT, having a low number of affected sites, and being female.

Key Words: peptide receptor radionuclide therapy; somatostatin analogs; neuroendocrine tumors; radiopharmaceutical; individualized therapy; long-term follow-up

J Nucl Med 2019; 60:524-529

DOI: 10.2967/jnumed.118.215376

\footnotetext{
Received May 29, 2018; revision accepted Aug. 10, 2018.

For correspondence contact: Michael Gabriel, Department of Nuclear Medicine, Medical University of Innsbruck, Anichstrasse 35, 6020 Innsbruck, Austria.

E-mail: michael.gabriel@kepleruniklinikum.at

Published online Aug. 16, 2018.

COPYRIGHT @ 2019 by the Society of Nuclear Medicine and Molecular Imaging.
}

$\mathbf{T}$ he expression of somatostatin receptors by neuroendocrine tumors (NETs) is the basis for peptide receptor radionuclide therapy (PRRT) $(1,2)$. Several, mainly $\beta$-radiation-emitting, compounds labeled with different somatostatin analogs are used for this systemic treatment approach in patients with metastatic or inoperable progressive disease (3). Depending on the size of the tumor or metastasis, ${ }^{90} \mathrm{Y} \beta$-rays with a range of approximately $12 \mathrm{~mm}$ in tissue are theoretically better suited for larger tumor lesions, whereas ${ }^{177} \mathrm{Lu}$, with a smaller range of approximately $2 \mathrm{~mm}$, is preferentially used for smaller tumors. Although there is no evidence in the clinical setting, this concept has been widely applied in clinical practice for many years. In the last decade, the ${ }^{177} \mathrm{Lu}$-labeled compound, particularly, has found its way into clinical routine in view of its more favorable properties in terms of kidney toxicity.

The clinical efficacy of PRRT has been demonstrated in several clinical studies (4-6). The response rate summing up complete response, partial response, minor response, and stable disease is about $70 \%-80 \%$ for ${ }^{90}$ Y-DOTATOC and for ${ }^{177} \mathrm{Lu}$-DOTATATE $(5,6)$. In general, the prognosis of patients responding to PRRT is favorable, meaning that median time to progression is about 3-4 y. However, if progressive disease occurs early after PRRT, the prognosis is poor $(5,0)$.

Despite these findings, not much is known about long-term outcome. Although this therapy approach has been available for about $20 \mathrm{y}$, experience with long-term outcome is limited. Only recent prospective study results have indicated a survival benefit as compared with established therapy procedures (7). Especially for metastatic midgut NETs, PRRT has been established as one major therapy strategy since only few therapeutic alternatives, with sometimes more severe side effects, are available for this tumor entity (8). But also for pancreatic NET, both of the available radiopharmaceuticals used for PRRT have shown advantages over other treatment approaches with regard to progression-free and overall survival (9).

On the other hand, PRRT also entails side effects that should also be considered in order to grasp the value of this oncologic therapy. In particular, special attention has to be paid to renal function and bone marrow reserve (10). In 2010, the Innsbruck group published its experience with a more individualized treatment scheme (11). It was found that PRRT with differently labeled tracers $\left({ }^{90} \mathrm{Y}\right.$ or $\left.{ }^{177} \mathrm{Lu}\right)$ and different somatostatin analogs was generally well tolerated, with only a few serious side effects. In particular, extended time intervals and reduced individual doses were proposed in patients with advanced tumor stages, in patients with moderate somatostatin receptor expression, and in patients of higher age. This 
TABLE 1

Age of Deceased Patients at Date of Death or of Survivors on Final Date of Evaluation

\begin{tabular}{llllrcc}
\hline \multicolumn{1}{c}{ Sex } & Life status & $n$ & Mean & SD & Minimum & Maximum \\
\hline \multirow{2}{*}{ Female } & Alive & 8 & 55.4 & 6.2 & 47 & 64 \\
& Dead & 9 & 62.1 & 13.2 & 41 & 78 \\
\multirow{4}{*}{ Male } & Alive & 6 & 56.2 & 6.4 & 47 & 62 \\
& Dead & 21 & 62.0 & 12.7 & 40 & 84 \\
All & Alive & 14 & 55.7 & 6.0 & 47 & 64 \\
& Dead & 30 & 62.0 & 12.6 & 40 & 84 \\
& Both & 44 & 60.0 & 11.3 & 40 & 84 \\
\hline
\end{tabular}

treatment regime also included retreatment in cases of progression. However, long-term experience with this concept is still not available. Thus, the aim of the present analysis was to document the medical history of patients initially treated with PRRT at our department more than $12 \mathrm{y}$ ago.

\section{MATERIALS AND METHODS}

The included patients were largely the same as published in The Journal of Nuclear Medicine in 2009 (12) thanks to the well-documented patient history after many years. Accordingly, tumors originated from neuroendocrine tissue of the gastrointestinal tract in 41 patients. In addition, 3 patients had a carcinoid tumor of the lung, and 1 patient each had a glomus tumor and a dendritic reticulum cell sarcoma. With a positive ${ }^{68} \mathrm{Ga}$-DOTATOC PET/CT result, patients were consecutively treated with ${ }^{90}$ Y-DOTATOC $(n=24),{ }^{177} \mathrm{Lu}-$ DOTATATE $(n=19)$, or both $(n=3)$ (12). Dosimetry was also performed using either the ${ }^{111}$ In-labeled compound before the first treatment cycle of ${ }^{90}$ Y-DOTATOC or using ${ }^{177} \mathrm{Lu}$-DOTATATE during the first therapy with special consideration of the kidney dose with the well-known threshold of $23 \mathrm{~Gy}$, which was taken from external-beam radiation (13). PET/CT was used for initial assessment and for restaging (12) and also formed the basis for analyzing quantitative parameters. The local ethics committee approved the initial study, and all

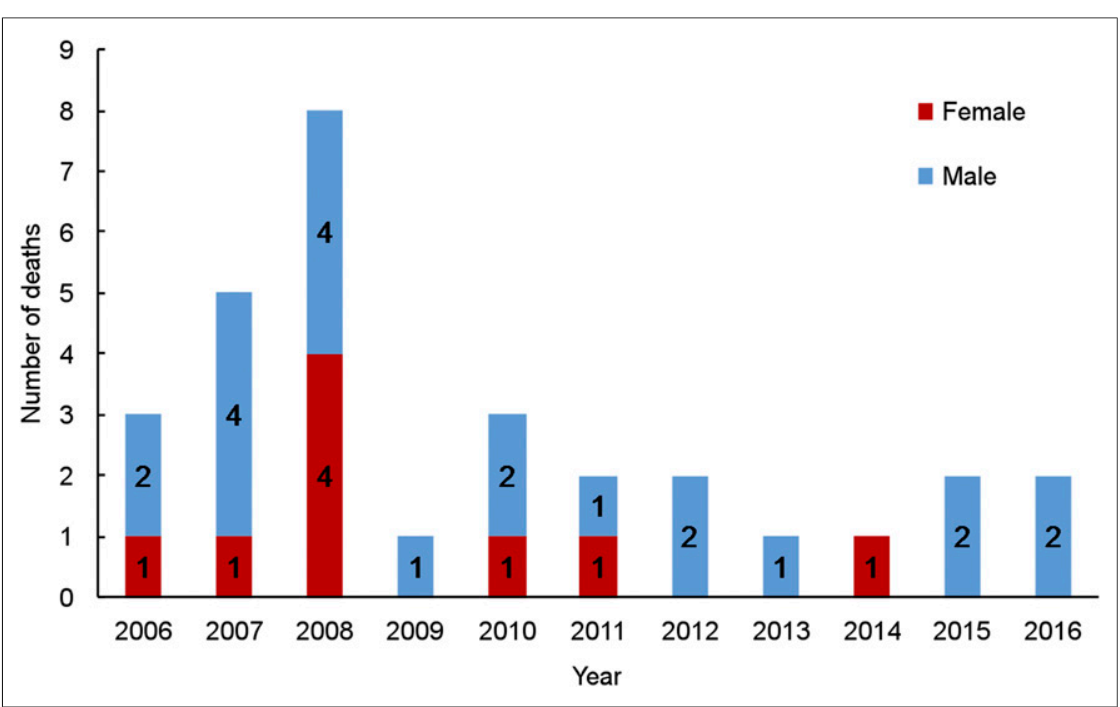

FIGURE 1. Graph showing number of deaths in various years. Of female patients, 3 died after 2008. subjects gave written informed consent. Two men ( 34 and 47 y old) were lost to follow-up and thus removed from the final analysis. One of these patients had a NET of the pancreas and the other a NET of the small bowel. The present reevaluation thus included a total of 27 (61\%) men and 17 (39\%) women. Mean age at initial diagnosis was $60.0 \mathrm{y}$ (SD, 11.3), with the youngest person being $40 \mathrm{y}$ and the oldest $84 \mathrm{y}$ at the time of initial diagnosis. Details are given in Table 1. The shortest duration of illness was 8 mo in a 61-y-old man who died of pneumonia; the longest was 155 mo in a 60 -y-old woman with a pancreatic NET and liver metastases. This patient is still in excellent clinical condition.

In contrast to the previous publication, which focused on the imaging method used, we here attempted to retrospectively evaluate the longterm outcome of these patients after they received PRRT according to our individualized treatment protocol as published earlier (11). For the following retrospective investigation, no separate ethical approval and no formal consent had to be obtained according to Austrian law.

Median overall survival, meaning the time after which $50 \%$ of patients were still living and $50 \%$ had died, included the time from the initial examination by ${ }^{68} \mathrm{Ga}$-DOTATOC PET/CT to the date of death or, for survivors, the last day of evaluation, namely November 4, 2017. Median survival refers to how long (mo) patients survived with a disease. In particular, it was the time when half the patients were expected to be alive.

Because of the long follow-up, it was found that intra- and interindividual follow-up controls significantly differed, so that assessment of progression-free survival was not feasible.

Median follow-up was 80 mo (first quartile to third quartile, $\mathrm{Q}_{1}-\mathrm{Q}_{3}$, 27.5-136.5 mo) for the whole population, whereas median follow-up was $139.5 \mathrm{mo}\left(\mathrm{Q}_{1}-\mathrm{Q}_{3}, 137-146 \mathrm{mo}\right)$ for survivors and $42.5 \mathrm{mo}\left(\mathrm{Q}_{1}-\right.$ $\mathrm{Q}_{3}, 23-81 \mathrm{mo}$ ) for patients who had already died.

A mean of $5.3 \pm 2.5$ cycles were administered for ${ }^{177} \mathrm{Lu}-\mathrm{PRRT}$, with mean activity of $27.2 \pm 14.9 \mathrm{GBq}$ per patient, and a mean of $5.5 \pm$ 2.6 cycles were administered for ${ }^{90}$ Y-PRRT, with mean activity of $14.7 \pm 7.3 \mathrm{GBq}$. Overall, 378 cycles were administered (mean, $8.6 \pm 3.4$ cycles per patient; range, 2-17 cycles), with an overall cumulative activity of 1,514.1 GBq (mean, $34.4 \pm 13.9 \mathrm{GBq}$ ).

\section{Imaging and Quantitative Assessment}

Patient imaging and image reconstruction were performed on a dedicated PET scanner (Advance; GE Healthcare) as described elsewhere (14). Irregular isocontour regions of interest were drawn over the target lesion at $50 \%$ of maximum pixel value within the tumor. The lesion that was most visible and easy to define was considered the index or target lesion. The individual patient's region of interest was placed in the same target lesion on the pre- and posttherapy PET scans for quantitative intrapatient comparison (12). Lesions with a diameter below 4 $\mathrm{cm}$, tumor lesions with increased tracer uptake on the rim, and lesions with no uptake in the center because of necrosis were excluded from quantitative evaluation to avoid pitfalls caused by the partial-volume effect.

$\mathrm{SUV}_{\text {max }}$ was calculated using the maximum activity in the region of interest normalized for the injected dose and patient body weight. Additionally, $\mathrm{SUV}_{\max }$ before and after initial PRRT and relative changes in $\mathrm{SUV}_{\text {max }}$ were analyzed for the target lesion; further details are described elsewhere (12). In 16 patients, individual target lesions decreased significantly in size, indicating remission to therapy. However, most patients 


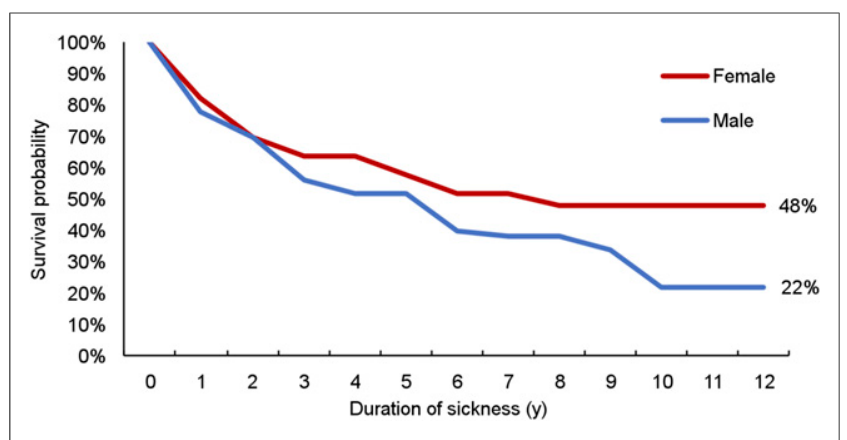

FIGURE 2. Life-span curves showing clear sex difference, but with log-rank test giving $P$ value of 0.195 .

showed no significant change in the index lesion from the visual aspect. Median $\mathrm{SUV}_{\max }$ before and after were $32.2\left(\mathrm{Q}_{1}-\mathrm{Q}_{3}, 23-44.5\right)$ and $25.6\left(\mathrm{Q}_{1}-\mathrm{Q}_{3}, 16.3-37.6\right)$, respectively. Median relative change in $\mathrm{SUV}_{\text {max }}$ was $-13.5 \%\left(\mathrm{Q}_{1}-\mathrm{Q}_{3},-49.4\right.$ to 16.6$)$.

\section{Treatment Schedule and Evaluation of Outcome}

In our concept (11), the ${ }^{90} \mathrm{Y}$-labeled compound was normally used as the first choice. However, if most individual lesions were smaller than $2 \mathrm{~cm}$ in diameter, ${ }^{177} \mathrm{Lu}$-DOTATATE was preferred for initial administration. For PRRT retreatment we normally used the ${ }^{177} \mathrm{Lu}-$ labeled compound.

Evaluation of treatment response and side effects was described in the aforementioned publications $(11,12)$. Adverse effects of therapy, such as every change in blood test results, were recorded and analyzed with the World Health Organization side effect scoring system for reporting results of cancer treatment. For health condition reporting, the Karnofsky Performance Index (15) was documented in each patient during every stay at the nuclear medicine department.

\section{Therapy After Completion of PRRT Beyond Radionuclide Therapy}

In addition to the application of cold long-acting somatostatin analogs in most patients $(n=27)$, a further 2 patients each were treated with chemotherapy and radiofrequency ablation. One patient with a pulmonary carcinoid received temozolomide, and one with a rectal NET received temozolomide and everolimus. One of the 2 patients treated with radiofrequency ablation of progredient liver metastases turned out to also have an exocrine tumor of the pancreatic head as a second malignant disease. After surgical removal of this tumor and radiochemotherapy with gemcitabine, this patient was considered cured with regard to this non-NET. The second patient undergoing radiofrequency ablation additionally received external-beam radiation of bone metastases in the lumbar spine, caused by a pancreatic NET.

\section{Data Collection and Statistical Data Analysis}

Patient and disease-related data were collected from hospital electronic records and imaging and from the medical reports of outside hospitals. In addition to the descriptive part of the analysis with special consideration of the median overall survival, the available data were also used to statistically analyze relationships to dependent factors, such as type of primary tumor, sex distribution, type and number of organ systems involved, kind of first radiopharmaceutical used for PRRT, initial response to therapy, and $\mathrm{SUV}_{\max }$ of reference lesions.

Statistical analysis was performed using R, version 3.3.2 (https:// www.r-project.org). Graphs and tables were created using Microsoft Excel and Microsoft Word.

Lifetime analyses were used to investigate differences in predefined groups. Kaplan-Meier graphs showed the probability of survival. The log-rank test was used to compare survival distribution between groups. The semiparametric Cox proportional-hazards model was used to test the influence of quantitative variables on survival, especially $\mathrm{SUV}_{\max }$ before PRRT and relative changes. In all analyses, a $P$ value of less than 0.05 was considered statistically significant.

\section{RESULTS}

\section{General Population}

As also shown in Table 1, $21(77.8 \%)$ of the 27 men and 9 (52.9\%) of the 17 women died in the meantime. Median overall survival was 79 mo (95\% confidence interval, 41-125 mo). The median survival was 75 mo for men and 107 mo for women. As of this writing, only 3 women have died after the first 3 y of observation, thus indicating a survival benefit for women, as indicated in Figures 1 and 2, without reaching statistical significance $(P=$ $0.195, \log$-rank test). Of the overall 30 patients who died, 25 died from tumor progression. Only one 84-y-old patient died from renal failure. In addition, 1 male patient each died from acute myeloblastic leukemia, cerebral hemorrhage, pneumonia, and a grand mal seizure unrelated to PRRT.

No statistical significance was observed when the site of the primary tumor was analyzed, as shown in Table $2(P=0.473, \log$ rank test).

When the number of affected organ systems was considered, it was found that $41.7 \%$ of the patients with only 1 system affected were still alive, whereas such was the case in only $27.3 \%$ of those involving 3 or 4 systems (Table 3 ). When median survival was compared between patients with 1 or 2 affected organ systems and patients with more extended disease, a difference could also be assumed, namely 97 or 81 mo versus 23 mo. Despite a clear tendency, no statistically significant difference could be observed when analyzing survival probability $(P=0.192, \log$ rank test).

TABLE 2

Analysis with Regard to Type of Primary Tumor

\begin{tabular}{lccccc}
\hline \multicolumn{1}{c}{ Primary tumor } & Alive $(n)$ & Dead $(n)$ & Alive (\%) & Mean age at diagnosis (y) & Median survival (mo) \\
\hline NET of pancreas & 4 & 12 & 25.0 & $59.3(\mathrm{SD}, 11.9)$ & $42(95 \% \mathrm{Cl}, 23-107)$ \\
\hline NET of small bowel & 6 & 9 & 40.0 & $58.1(\mathrm{SD}, 10.6)$ & $125(95 \% \mathrm{Cl}, 30-\mathrm{NA})$ \\
NET unknown primary & 2 & 2 & 50.0 & $59.8(\mathrm{SD}, 10.4)$ & $\mathrm{NA}(95 \% \mathrm{Cl}, 75-\mathrm{NA})$ \\
NET of lung & 1 & 3 & 25.0 & $65.8(\mathrm{SD}, 5.9)$ & $49(95 \% \mathrm{Cl}, 8-\mathrm{NA})$ \\
NET of rectum & 1 & 2 & 33.3 & $67.0(\mathrm{SD}, 17.0)$ & $115(95 \% \mathrm{Cl}, 20-\mathrm{NA})$ \\
Reticulum sarcoma, glomus tumor & 0 & 2 & 0.0 & $58.5(\mathrm{SD}, 20.5)$ & $51.0(95 \% \mathrm{Cl}, 23-79)$ \\
\hline
\end{tabular}


TABLE 3

Analysis According to Number of Affected Organ Systems

\begin{tabular}{lccccc}
\hline No. of systems & Alive $(n)$ & Dead $(n)$ & Alive (\%) & Mean age at diagnosis (y) & Median survival (mo) \\
\hline 1 & 5 & 7 & 41.7 & $60.8(\mathrm{SD}, 9.4)$ & $97(95 \% \mathrm{Cl}, 23-\mathrm{NA})$ \\
2 & 6 & 15 & 28.6 & $60.0(\mathrm{SD}, 10.6)$ & $81(95 \% \mathrm{Cl}, 41-127)$ \\
3,4 & 3 & 8 & 27.3 & $59.3(\mathrm{SD}, 14.8)$ & $23(95 \% \mathrm{Cl}, 18-\mathrm{NA})$ \\
& & & & & \\
\hline $\mathrm{NA}=$ not applicable. & & & & & \\
\hline
\end{tabular}

\section{Patients Alive at Date of Censoring}

Fourteen patients were still alive more than $12 \mathrm{y}$ after initial PRRT, namely 8 women and 6 men. The final evaluation showed stable disease in 7 patients, progressive disease in 5, and partial response in 2 .

Of the 14 patients still alive, 13 received additional cycles of PRRT during the course of disease, that is, retreatment when disease progression was observed: 8 with ${ }^{177}$ Lu-DOTATATE, 2 with ${ }^{90}$ Y-DOTATOC, and 3 with ${ }^{177} \mathrm{Lu}$-DOTATATE and ${ }^{90}$ Y-DOTATOC. The highest activities applied to these patients were $54.93 \mathrm{GBq}$ for ${ }^{177} \mathrm{Lu}$-DOTATATE, $27 \mathrm{GBq}$ for ${ }^{90} \mathrm{Y}$-DOTATOC, and $50 \mathrm{GBq}$ for the combined use of both treatment regimes $\left(35.4 \mathrm{GBq}\right.$ of ${ }^{177} \mathrm{Lu}-$ DOTATATE and $14.6 \mathrm{GBq}$ of ${ }^{90}$ Y-DOTATOC). These 13 patients also received long-acting somatostatin analogs.

Most of these patients were in good clinical condition, with a Karnofsky Performance Index of $100 \%$ in 8 patients, $90 \%$ in 3 , $80 \%$ in 2 , and $70 \%$ in only one 61 -y-old man who suffered from chronic anemia, diarrhea, and grade IV renal toxicity.

\section{Side Effects in Patients Alive at Date of Censoring}

Overall, severe side effects (World Health Organization grades III and IV) were seen in $9(64 \%)$ of 14 patients. Severe long-term nephrotoxicity (grades III and IV) in combination with anemia was the most common finding in these patients. Furthermore, 1 patient had grade II nephrotoxicity and anemia. None of the remaining 4 patients showed nephrotoxicity. However, 1 of these patients suffered from chronic diarrhea, 1 from moderate anemia, and 1 from thrombopenia.

\section{Evaluation of Therapy Response and Factors Predicting Outcome}

Patients who responded well to the first PRRT in terms of stabilizing the underlying disease or showing remission had a significantly better survival probability than did those who presented with tumor progression after initial therapy $(P<0.001)$, as also shown in Table 4 and Figure 3.
A statistically significant result was also obtained for the choice of radiopharmaceutical used for therapy, meaning that patients treated with ${ }^{177} \mathrm{Lu}$-DOTATATE had a significantly better survival probability than did those treated with ${ }^{90}$ Y-DOTATOC $(P<$ 0.001), as also depicted in Figure 4. Nevertheless, many of the patients were also switched to the other radiopharmaceutical during the further course of the disease.

No significant relationship was found between the duration of disease in months and the quantitative parameter $\mathrm{SUV}_{\max }$ before PRRT $(P=0.597)$ or relative changes in $\operatorname{SUV}_{\max }(P=0.328)$.

\section{DISCUSSION}

Two decades after the introduction of PRRT, the long-term results are becoming more and more interesting. Only a few reports are presently available, and they give an impression of long-term efficacy, survival, and safety of PRRT. In our patient group, median overall survival from the beginning of the first PRRT cycle was 79 mo. Overall, $21(77.8 \%)$ of the 27 men and $9(52.9 \%)$ of the 17 women died, which means that nearly one third of the treated patients were still alive more than $12 \mathrm{y}$ after initial diagnosis of inoperable metastatic disease. Different studies report median overall survival ranging from 22 to 71 mo $(4,16-20)$.

This longer median overall survival in our patient population might be attributed to our applied concept based on the repeated use of radiopharmaceuticals in the event of recurrent progression of the underlying disease using individually adapted activities (11). Forrer et al. also showed that ${ }^{177} \mathrm{Lu}$-DOTATOC therapy is feasible, safe, and efficacious in patients with relapse after ${ }^{90} \mathrm{Y}-$ DOTATOC treatment (2). After all, 13 of the 14 surviving patients also received additional cycles of PRRT after the initial therapy and thus exceeded the dosimetrically calculated kidney doses by several times.

On the other hand, the surviving patients in our study population had a large proportion of serious side effects possibly related to PRRT. Nine of the 14 patients still alive have grades III and IV side

TABLE 4

Analysis in Terms of Initial Response to Therapy and Median Overall Survival

\begin{tabular}{lccccc}
\hline \multicolumn{1}{c}{ Response to therapy } & Alive $(n)$ & Dead $(n)$ & Alive (\%) & Mean age at diagnosis (y) & Median survival (mo) \\
\hline Partial or complete response & 3 & 7 & 30.0 & $65.6(\mathrm{SD}, 10.7)$ & $86(95 \% \mathrm{Cl}, 23-\mathrm{NA})$ \\
Stable disease or minor response & 11 & 14 & 44.0 & $57.2(\mathrm{SD}, 10.2)$ & $125(95 \% \mathrm{Cl}, 79-\mathrm{NA})$ \\
Progressive disease & 0 & 9 & 0.0 & $61.6(\mathrm{SD}, 13.2)$ & $25(95 \% \mathrm{Cl}, 16-46)$ \\
& & & & & \\
\hline $\mathrm{NA}=$ not applicable. & & & & \\
\hline
\end{tabular}




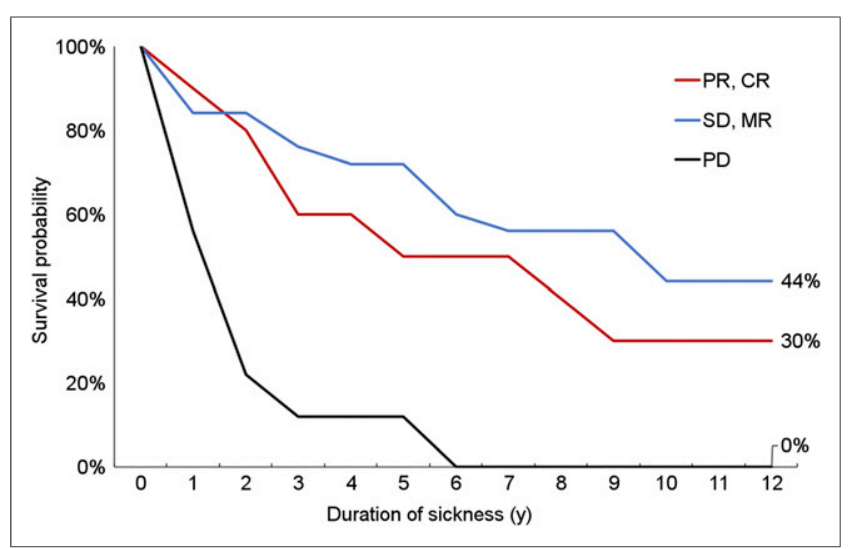

FIGURE 3. Kaplan-Meier curves clearly indicating that patients who showed cumulative disease control (complete response [CR] + partial response $[\mathrm{PR}]+$ minor response $[\mathrm{MR}]+$ stable disease $[\mathrm{SD}]$ ) after initial PRRT regime had significantly better overall survival than did patients with progressive disease (PD) $(P<0.001$, log rank).

effects; severe chronic renal toxicity in combination with anemia was the most common finding in these patients. This high rate of serious side effects is significantly higher than in the previously published studies (21). Even in those 7 patients who were treated throughout with ${ }^{177}$ Lu-DOTATATE, 3 developed severe nephrotoxicity. Nevertheless, none of the 14 patients were dialysis-dependent and most of the patients showed a still high Karnofsky Performance Index, which underlines the positive effect of PRRT in terms of the quality of life (22).

In contrast to the observation made by other investigators $(23,24)$, we found no correlation between outcome and quantitative parameters of tracer accumulation by applying $\mathrm{SUV}_{\max }$ and relative changes in $\mathrm{SUV}_{\text {max }}$. This finding could be related to our retrospective design but could also be a matter of insufficient assessment of somatostatin receptor distribution in tumor lesions. A recent publication, for instance, identified 8 statistically independent heterogeneity parameters for time to progression and time to death, whereas conventional PET parameters failed in response prediction (25).

An important indicator of median overall survival is the cumulative disease control to therapy after the first PRRT, especially in the patients who showed early progression had a poor prognosis. The subgroups with remission or stable disease showed no significant difference in outcome. Therefore, this feature is a predictor not only for midterm outcome but also for the long-term course over many years, as already previously assumed $(5,26)$. Consequently, at least stabilization of the underlying disease can be defined as a major therapeutic goal with important implications for further prognosis.

Although no significant difference could be identified, the Kaplan-Meier survival plots possibly indicated a survival benefit with regard to the female sex. Because there was no apparent difference in age, diagnosis, disease spread, or the therapeutic methods used, it can be assumed that this benefit is an inherent effect. It is interesting that of the female patients, only 3 died after 2008. Whether this potential survival benefit of women is due to better response to PRRT or whether women with a metastatic NET have a better survival benefit remains an unanswered question (27).

As shown in Table 3, when comparing the group of patients with 1,2 , or more affected organ systems at initial diagnosis, we found a difference with regard to the percentage of patients presently alive,

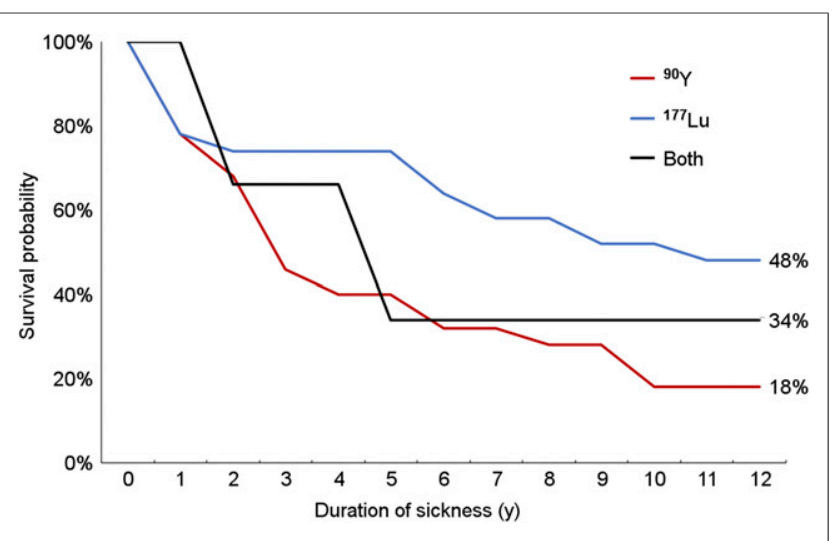

FIGURE 4. Graph showing that only $18 \%$ of patients initially treated with ${ }^{90}$-therapy were alive after $12 \mathrm{y}$, whereas nearly $48 \%$ of patients in ${ }^{177}$ Lu group were alive.

but this difference did not reach significance. This observation is underlined by considering the mean duration of median survival of each patient group. Patients with 1 or 2 affected organs showed a median survival of 97 and $81 \mathrm{mo}$, respectively, whereas in patients with more widespread disease, that is, 3 or more affected organs, median survival was 23 mo. This observation could be based on differences in tumor aggressiveness, meaning that more aggressive tumors tend to spread more widely whereas less biologically aggressive tumors show a more limited disease spread. Another analysis also confirms the favorable response and long-term outcome in patients who were treated in a phase of early progression rather than in a state of overt progression with higher tumor load (28).

According to the present analysis, the initial use of ${ }^{177} \mathrm{Lu}$ has shown significantly better results than has the use of ${ }^{90} \mathrm{Y}$ or the combination of both tracers, thus obviously supporting the present prevalent concept of exclusive use of ${ }^{177} \mathrm{Lu}$-DOTATATE for PRRT. However, one should also bear in mind that the choice of radiopharmaceutical used for the initial treatment regime in our patient population was largely related to the size of the individual tumor lesion as depicted by PET/CT. Considering the complex situation including also the tumor dose and volume in different-sized lesions (29), the present analysis should be cautiously interpreted; combined use with ${ }^{90} \mathrm{Y} /{ }^{177} \mathrm{Lu}$-DOTATATE was basically found to be highly effective, safe and showed similar results $(30,31)$.

Our study had 2 limitations. First, it used a retrospective design and assessed a relatively small number of patients in a single institution. Therefore, our results may have been affected by selection bias, as also mentioned above with regard to the choice of radiopharmaceutical. Second, histopathology of the included patients was rather heterogeneous in terms of the primary site, and grading a tumor-based analysis was not feasible, as indicated in Table 2.

\section{CONCLUSION}

Treatment with radiolabeled somatostatin analogs in this study was encouraging in terms of long-term outcome, as $32 \%$ of patients (14/44) with metastatic or inoperable disease were still alive more than $12 \mathrm{y}$ after the beginning of radionuclide therapy. Possible predictors for favorable outcome are initial response to PRRT, number of affected sites, and the sex of the patient. Despite chronic kidney disease in most surviving patients, none have 
needed dialysis and all have remained in good overall clinical condition.

\section{DISCLOSURE}

No potential conflict of interest relevant to this article was reported.

\section{REFERENCES}

1. Jong M, Breeman WA, Kwekkeboom DJ, Valkema R, Krenning EP. Tumor imaging and therapy using radiolabeled somatostatin analogues. Acc Chem Res. 2009;42:873-880.

2. Forrer F, Uusijärvi H, Storch D, Maecke HR, Mueller-Brand J. Treatment with ${ }^{177} \mathrm{Lu}$-DOTATOC of patients with relapse of neuroendocrine tumors after treatment with ${ }^{90}$ Y-DOTA-TOC. J Nucl Med. 2005;46:1310-1316.

3. van Essen M, Krenning EP, Kam BL, de Jong M, Valkema R, Kwekkeboom DJ. Peptide-receptor radionuclide therapy for endocrine tumors. Nat Rev Endocrinol. 2009;5:382-393.

4. Kwekkeboom DJ, de Herder WW, Kam BL, et al. Treatment with the radiolabeled somatostatin analog $\left[{ }^{177} \mathrm{Lu}-\mathrm{DOTA}^{0}, \mathrm{Tyr}^{3}\right]$ octreotate: toxicity, efficacy, and survival. J Clin Oncol. 2008;26:2124-2130.

5. Kwekkeboom DJ, Kam BL, van Essen M, et al. Somatostatin-receptor-based imaging and therapy of gastroenteropancreatic neuroendocrine tumors. Endocr Relat Cancer. 2010;17:R53-R73.

6. Kwekkeboom DJ, de Herder WW, van Eijck $\mathrm{CH}$, et al. Peptide receptor radionuclide therapy in patients with gastroenteropancreatic neuroendocrine tumors. Semin Nucl Med. 2010;40:78-88.

7. Strosberg J, El-Haddad G, Wolin E, et al. Phase 3 trial of ${ }^{177}$ Lu-Dotatate for midgut neuroendocrine tumors. N Engl J Med. 2017;376:125-135.

8. Strosberg JR, Halfdanarson TR, Bellizzi AM, et al. The North American Neuroendocrine Tumor Society consensus guidelines for surveillance and medical management of midgut neuroendocrine tumors. Pancreas. 2017;46:707-714.

9. Prasad V, Horsch D, Hommann M, Zachert C, Baum RP. Survival benefits and efficacy of peptide receptor radionuclide therapy (PRRT) using Y-90/Lu-177 DOTA-TATE in pancreatic neuroendocrine tumors (pNET) [abstract]. $J$ Nucl Med. 2009;50(suppl 2):43.

10. Bodei L, Kwekkeboom DJ, Kidd M, Modlin IM, Krenning EP. Radiolabelled somatostatin analogue therapy of gastroenteropancreatic cancer. Semin Nucl Med. 2016;46:225-238.

11. Gabriel M, Andergassen U, Putzer D, et al. Individualized peptide-related-radionuclide-therapy (PRRT) concept using different radiolabelled somatostatin (SST) analogs in advanced cancer patients. Q J Nucl Med Mol Imaging. 2010;54: 92-99.

12. Gabriel M, Oberauer A, Dobrozemsky G, et al. ${ }^{68}$ Ga-DOTA-Tyr3-octreotide PET for assessing response to somatostatin-receptor-mediated radionuclide therapy. J Nucl Med. 2009;50:1427-1434.

13. Emami B, Lyman J, Brown A, et al. Tolerance of normal tissue to therapeutic irradiation. Int J Radiat Oncol Biol Phys. 1991;21:109-122.

14. Gabriel M, Decristoforo C, Kendler C, et al. ${ }^{68}$ G-DOTA-Tyr ${ }^{3}$-octreotide PET in neuroendocrine tumors: comparison with somatostatin receptor scintigraphy and CT. J Nucl Med. 2007;48:508-518.

15. Karnofsky DA, Burchenal JH. The clinical evaluation of chemotherapeutic agents in cancer. In: MacLeod CC, ed. Evaluation of Chemotherapeutic Agents in Cancer. New York, NY: Columbia University Press; 1949:191-205.
16. Valkema R, Pauwels S, Kvols LK, et al. Survival and response after peptide receptor radionuclide therapy with $\left[{ }^{90} \mathrm{Y}\right.$-DOTA0,Tyr $\left.{ }^{3}\right]$ octreotide in patients with advanced gastroenteropancreatic neuroendocrine tumors. Semin Nucl Med. 2006;36:147-156.

17. Bushnell DL Jr, O'Dorisio TM, O'Dorisio MS, et al. ${ }^{90}$ Y-edotreotide for metastatic carcinoid refractory to octreotide. J Clin Oncol. 2010;28:1652-1659.

18. Cwikla JB, Sankowski A, Seklecka N, et al. Efficacy of radionuclide treatment DOTATATE Y-90 in patients with progressive metastatic gastroenteropancreatic neuroendocrine carcinomas (GEP-NETs): a phase II study. Ann Oncol. 2010;21: 787-794.

19. Brabander T, van der Zwan WA, Teunissen JJM, et al. Long-term efficacy, survival, and safety of $\left[{ }^{177} \mathrm{Lu}-\mathrm{DOTA}{ }^{0}, \mathrm{Tyr}^{3}\right]$ octreotate in patients with gastroenteropancreatic and bronchial neuroendocrine tumors. Clin Cancer Res. 2017;23: 4617-4624.

20. Vaughan E, Machta J, Walker M, Toumpanakis C, Caplin M, Navalkissoor S. Retreatment with peptide receptor radionuclide therapy in patients with progressing neuroendocrine tumours: efficacy and prognostic factors for response. $\mathrm{Br} \mathrm{J}$ Radiol. 2018;20:20180041.

21. van der Zwan WA, Bodei L, Mueller-Brand J, de Herder WW, Kvols LK, Kwekkeboom DJ. Radionuclide therapy in neuroendocrine tumors. Eur J Endocrinol. 2015;172:R1-R8.

22. Öksüz MÖ, Winter L, Pfannenberg C, et al. Peptide receptor radionuclide therapy of neuroendocrine tumors with ${ }^{90}$ Y-DOTATOC: is treatment response predictable by pre-therapeutic uptake of ${ }^{68} \mathrm{Ga}$-DOTATOC? Diagn Interv Imaging. 2014;95:289-300.

23. Khan S, Krenning EP, van Essen M, Kam BL, Teunissen JJ, Kwekkeboom DJ. Quality of life in 265 patients with gastroenteropancreatic or bronchial neuroendocrine tumors treated with $\left[{ }^{177} \mathrm{Lu}-\mathrm{DOTA}{ }^{0}, \mathrm{TYR}^{3}\right]$ octreotate. J Nucl Med. 2011;52: 1361-1368.

24. Haug AR, Auernhammer CJ, Wängler B, et al. ${ }^{68}$ Ga-DOTATATE PET/CT for the early prediction of response to somatostatin receptor-mediated radionuclide therapy in patients with well-differentiated neuroendocrine tumors. J Nucl Med. 2010;51: 1349-1356.

25. Werner RA, Lapa C, Ilhan H, et al. Survival prediction in patients undergoing radionuclide therapy based on intratumoral somatostatin-receptor heterogeneity. Oncotarget. 2017;8:7039-7049.

26. Hamiditabar M, Ali M, Roys J, et al. Peptide receptor radionuclide therapy with ${ }^{177} \mathrm{Lu}$-octreotate in patients with somatostatin receptor expressing neuroendocrine tumors: six years' assessment. Clin Nucl Med. 2017;42:436-443.

27. Hallet J, Law CH, Cukier M, Saskin R, Liu N, Singh S. Exploring the rising incidence of neuroendocrine tumors: a population-based analysis of epidemiology, metastatic presentation, and outcomes. Cancer. 2015;121:589-597.

28. Ezziddin S, Attassi M, Yong-Hing CJ, et al. Predictors of long-term outcome in patients with well-differentiated gastroenteropancreatic neuroendocrine tumors after peptide receptor radionuclide therapy with ${ }^{177}$ Lu-octreotate. J Nucl Med. 2014;55: $183-190$.

29. Ilan E, Sandström M, Wassberg C, et al. Dose response of pancreatic neuroendocrine tumors treated with peptide receptor radionuclide therapy using ${ }^{177} \mathrm{Lu}-$ DOTATATE. J Nucl Med. 2015;56:177-182.

30. Kunikowska J, Pawlak D, Bak MI, Kos-Kudla B, Mikolajczak R, Krolicki L. Long-term results and tolerability of tandem peptide receptor radionuclide therapy with ${ }^{90} \mathrm{Y} /{ }^{177} \mathrm{Lu}$-DOTATATE in neuroendocrine tumors with respect to the primary location: a 10-year study. Ann Nucl Med. 2017;31:347-356.

31. Lorenzoni A, Capozza A, Artale S, Mascauro M, Sergni EC. Impressive response to tandem treatment with $\left[{ }^{90} \mathrm{Y}\right]$ DOTATOC and $\left[{ }^{177} \mathrm{Lu}\right]$ DOTATOC in grade 3 pancreatic neuroendocrine carcinoma. Clin Nucl Med. 2018;43:506-508.

\section{Erratum}

In the article "In Vivo PET Imaging Demonstrates Diminished Microglial Activation After Fingolimod Treatment in an Animal Model of Multiple Sclerosis," by Airas et al. (J Nucl Med. 2015;56:305-310), author Susanne Vainio was inadvertently left out of the author and affiliation lists. The corrected author line should read: Airas L, Dickens AM, Elo P, Marjamäki, P, Johansson J, Vainio S, Eskola O, Jones PA, Trigg W, Solin O, Haaparanta-Solin M, Anthony DC, and Rinne J. Dr. Vainio's affiliation should have been listed as: Turku PET Centre Preclinical Imaging, Turku, Finland. The authors regret the error. 